\title{
Vorwort des Autors und Koautors zum Buch „Klinische Hämostaseologie für Zahnärzte und Oralchirurgen ${ }^{66}$
}

Sehr geehrte Kolleginnen und Kollegen,

im vorliegenden Buch „Klinische Hämostaseologie für Zahnärzte und Oralchirurgen“ der Buchreihe „Blutgerinnung interdisziplinär“ möchten wir zahnärztlich, oralchirurgisch und auch kieferchirurgisch tätigen Zahnärzten und Ärzten klinisch relevante Aspekte der Hämostaseologie vermitteln. Hierbei legen wir Wert auf eine praxisorientierte Darstellung, die es ermöglichen soll, das eigene Wissen zu vertiefen und das Verständnis für das „Gerinnungsmanagement“ in diesem Fachgebiet zu vertiefen.

Eingangs wird der physiologische Prozess der Blutgerinnung dargestellt, dessen Kenntnis die Grundlage für das Verständnis der Gerinnungsdiagnostik und der Pathophysiologie von Gerinnungsstörungen darstellt. Angeschlossen werden Grundlagen $\mathrm{zu}$ Gerinnungsdefekten, die mit einer Blutungsneigung assoziiert sind. Zudem wird eine Übersicht über aktuelle antithrombotische Substanzen gegeben, da erfahrungsgemäß häufig eine erhebliche Unsicherheit besteht, wie mit diesen Pharmaka - also Antikoagulanzien und Plättchenfunktionshemmern - periinterventionell im Rahmen von zahnärztlichen und oralchirurgischen Eingriffen umzugehen ist.

Nachfolgend werden dann die für Zahnärzte und Oralchirurgen relevante Themenkomplexe aus dem Gebiet der Hämostaseologie gesondert dargestellt: Hierbei wird auf das Management bei Patienten mit vermehrter Blutungsneigung bzw. mit Gerinnungsdefekten, die mit einem erhöhten periinterventionellen Blutungsrisiko assoziiert sind, eingegangen. Des Weiteren gehen wir ausführlich auf die wachsende Zahl von Patienten mit thrombotischen und thromboembolischen Erkrankungen ein, die sich zahnärztlichen und oralchirurgischen Eingriffen unterziehen müssen. Hierbei wird ein besonderer Schwerpunkt auf das periinterventionelle Management bei Patienten, die Antikoagulanzien und/oder Plättchenfunktionshemmer einnehmen, gelegt.

Abschließend werden typische hämostaseologische Kasuistiken aus dem Gebiet der Zahnmedizin und Oralchirurgie dargestellt und hierbei auf relevante Fragestellungen und Lösungsansätze eingegangen. 
Im Rahmen der Erstellung des Manuskripts haben wir versucht, die für Zahnärzte und Oralchirurgen klinisch relevanten Aspekte aus dem Gebiet der Hämostaseologie herauszuarbeiten und somit dem Leser eine Vertiefung und Erweiterung des Wissens zu ermöglichen. Wir hoffen und wünschen uns, dass wir hierdurch einen Beitrag zur Optimierung des hämostaseologischen Managements im Rahmen zahnärztlicher und oralchirurgischer Eingriffe geleistet haben.

Berlin, Januar 2019

Christoph Sucker Claus Schüttler-Janikulla 